\title{
The Effects of Cold Therapy in Losing Weight \& Inches as well as Decreasing Body Mass Index, Body Fat Percentage, and Lowering of Health Risk Factor
}

\section{Ariel S Torres*}

Marie France Bodyline, Philippines

\begin{abstract}
Introduction: Many studies have been done to show that cold exposure increases calorie burning thru increased non-shivering thermogenesis (NST),increased putative Brown Adipose Tissue (BAT) activation and increasing the resting metabolic rate (RMR). But there has been no documentation on its end result of producing weight loss, inch loss, as well as decreased fat mass. Cold wraps have been used by slimming centre's since the 1960's to lose weight by thermogenesis and recently, they have converted to cold machine treatments.

Materials and Methods: A chronologic sample of 41,098 slimming treatments of 1,859 patients coming from 11 slimming centres in the Philippines with cold machine slimming programs consisting of 13,638 cold machine treatments were collated from November 4, 2014 to July 29, 2015. A random sample of 10 patients per centre that fit the inclusion \& exclusion criteria was then used. After applying the inclusion \& exclusion criteria together with the random sampling, only 1,028 cold machine treatments of 106 patients remained.

Results: The average weight loss per cold machine treatment by median is $0.5 \mathrm{lb}$. $(1.4583 \mathrm{lb}$. highest, -0.44 $\mathrm{lb}$. lowest), by mode range is $0.4-0.49 \mathrm{lb}$. and $0.5-0.59 \mathrm{lb}$. (16 \& 15), and by mean is $0.40 \mathrm{lb}$. (415.65 lbs./1,028 treatments). Regarding additional measurements and calculations done after completing 8-12 cold machine treatments, the average body mass index decreased by almost one $(0.77338) \mathrm{kg} / \mathrm{m} 2$ with $13 \%$ changing their BMI classification from obese to overweight and from overweight to normal weight. The average inch loss is slightly more than $1 \frac{1}{2}$ " (1.518 inches). Health risk factor was lowered in $32 \%$ with those in very high risk becoming just medium risk while those in medium risk becoming just very low risk. Using a body composition analysis machine(TANITA Impedance Method), it was noted that $77 \%$ decreased their total body fat percentage, $31 \%$ lowered their visceral fat rating, while $77 \%$ decreased the subcutaneous fat volume in their tummy area.

Conclusion: Cold treatment using cold machine mimics the same approximate weight loss result of the cold wraps $(0.5091 \mathrm{lb}$. vs. $0.5195 \mathrm{lb}$., respectively). It is an effective treatment for inducing weight loss and producing inch loss, as well as decreasing body mass index, body fat percentage, $\&$ health risk factor.
\end{abstract}

Keywords: Weight loss; Inch loss; Body mass index (BMI); Body Fat Percentage, Health Risk Factor; Cold machine treatments; Slimming treatments; Slimming programs; Brown Adipose Tissue; Non-shivering thermogenesis.

\section{Introduction}

The cold machine [1] treatment is a slimming therapy using a machine that cools water and pushes it into rubber tubing's arranged in an array like a blanket. This is then wrapped around the body in order to stimulate non-shivering thermogenesis (NST) to burn fat by activating putative Brown Adipose Tissue (BAT). It boasts of losing weight and inches with each succeeding treatment. Several studies [1,2] have been done to document the increase in resting metabolic rate (basal metabolism) thru indirect calorimetry and non-shivering thermogenesis with cold exposure. FDG-PET scans have proven not only presence of BAT but also their increased activity with cold exposure [1,3]. In turn, the activation of BAT have beneficial physiologic functions [4,5].

But even with proven increased calorie burning [1], there has been no documentation on its end result of producing weight loss, inch loss, as well as decreased fat mass. On the other hand, cold wraps have been used in Europe [6], specifically by slimming centres in Switzerland where it originated. Since the 1960's, they've used it to lose weight by thermogenesis and recently, they have converted to cold machine treatments. A local unpublished in-house study (1998) for cold wraps documented an average weight loss of $0.5195 \mathrm{lb}$. but no documentation on inch loss or decreased body fat mass.
The cold wrap has advantages: Of being very simple, cheap, readily available supplies (solution and bandage), and proven use for the last half century (since 1960's), easily adaptable to any body size (even very tall or very wide patient), mobility while having the treatment, and may even be taken out and done at home. The disadvantages are: Being messy with soaked solutions being wrapped around the body, archaic to the point of being primitive, colder at the start with sensation of chills, temperature not sustained once solution has evaporated, and prone to liquid-based or water-born infection (95\% of the solution is made up of water). The cold machine has advantages: Of having more control regarding maintenance of the target temperature (no shivering), with the hype of being high tech in this industrial period of computer era, and the skin is not exposed to wet conditions for prolonged periods of time. The disadvantages are: That the machine is expensive, the patient

${ }^{*}$ Corresponding author: Ariel Torres, M.D. Medical Doctor, Marie France Bodyline Int'l., Philippines, Tel: 632917489 2168; E-mail: yeltres@yahoo.com

Received November 2,2017;Accepted November 8, 2017; Published November 15, 2017

Citation: Torres AS (2017) The Effects of Cold Therapy in Losing Weight \& Inches as well as Decreasing Body Mass Index, Body Fat Percentage, and Lowering of Health Risk Factor. J Nutr Weight Loss 1: 107

Copyright: @ 2017 Torres AS. This is an open-access article distributed under the terms of the Creative Commons Attribution License, which permits unrestricted use, distribution, and reproduction in any medium, provided the original author and source are credited. 
needs to stay put and not move around (lying down), and not yet that established with regards to proven weight loss (no previous track record).

Recently, there has been emphasis on waist circumference (WC) as a better alternative for detecting increased visceral fat with concomitant increase in developing metabolic as well as cardiovascular diseases $[7,8]$. Obesity organizations are still using Body Mass Index (BMI) as a means of classifying if a person is suffering from obesity and to what degree. The advantage of BMI is that there's emphasis put on excess in total body weight (assumed to be body fat) to include subcutaneous fat, and not only visceral fat. The disadvantage of BMI is that muscles can be the reason for a high $\mathrm{BMI}$ and not necessarily fat. However, a combination of the two (BMI \& WC) is currently being used to determine the Health Risk Factor wherein general health is improved if BMI \& WC are both decreased at the same time $[9,10]$.

\section{Objective}

To determine if cold machine treatments can mimic the same weight loss of the cold wraps and gather additional data on improvements in inch loss \& specific fat loss.

\section{Subjects}

The cold machine1 treatment was introduced at private slimming centres in the Philippines during their 2014 anniversary month of November. Although it was already being beta-tested in one centre for several months, it was not offered to all patients. It only became commercially available for all patients and other centres in November of 2014. Initially it was only at the 4 biggest centres. The following month of December, it also became available to 4 additional mediumsized centres. By March of the following year, 2 more small-sized centres already had it. One remote slimming centre was the last to have it in May of 2015. All the patients from the time the machines became available at their respective centres that underwent cold machine treatment were included in this study from November 4, 2014 until July 29, 2015 when data was collected.

There was a total of 1,859 patients from 11 slimming centres that did 13,638 cold machine treatments with additional 27,460 other additional slimming treatments for a ratio of 1:2. Most were treated for weight loss coupled with inch loss and anti-cellulite treatments. A few were coupled with other machine treatments either on the day of their cold machine treatment or in between days. Such machines used the technologies of trans epidermal nerve stimulation, infra-red and red light, monopolar, bipolar, tripolar and multipolar radio frequency, thermo magnetic pulse, mechanical and thermal ultrasound, lipolysis via cryotherapy (freezing), and last are those that employ suction/ vacuum for skin curving (lymphatic drainage of fat). There were 1,696 female patients (91\%) and 163 male patients (9\%) for a M:F ratio of 1:10 representing all the centres (Figure 1 ). In the random sample study group, the age of patients ranged from 16 to 81 years old, from 4 ' $71 \frac{1}{2}$ " to 6' 0 1/2” " in height, from 107 lbs. -428 lbs. in weight, from normal weight to obese type III in BMI class, and from Very Low Risk to Very High Risk in Health Risk Factor. There were 81 female patients (82\%) and 18 male patients (18\%) for a M:F ratio of 1:4 (Figure 2) while the other 7 males were not included in computing the male to female ratio since they came from the slimming centre exclusively for males.

\section{Methodology}

Since any treatment effectively has its peaks (high) and valleys (low), a specific range for the number of cold machine treatments was

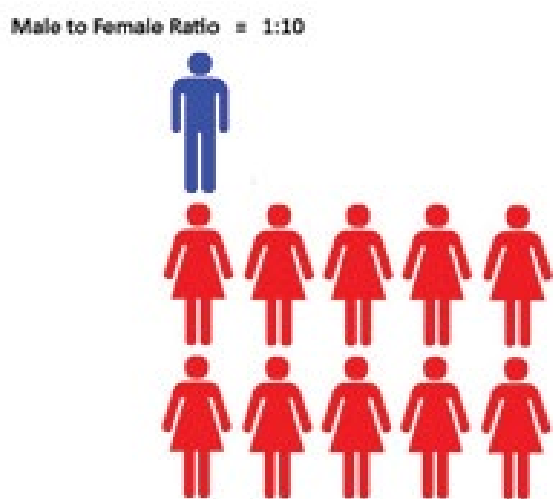

Figure 1: All the patients that underwent cold machine.

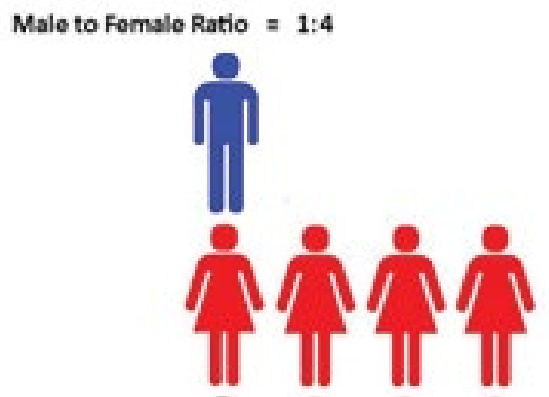

Figure 2: Random sample study group.

designated. During the first few treatments, the effect might not yet be evident so only those that had at least eight (8) cold machine treatments were included. On the other hand, the effect of the latter treatments might already be waxing and waning after several to numerous ones so data for those more than twelve (12) treatments were excluded. Due to the voluminous data, only a random sample was considered. The first 10 patients per centre randomly listed alphabetic of first name by information technology department (but no preference for gender, age, weight, height or dates of the cold machine program) that would fit the inclusion and exclusion criteria were used. The presence or absence of other slimming treatments would not matter in the choice for random sampling but if present, will be enumerated for data interpretation.

\section{Inclusion Criteria ${ }^{\star}$ for this study:}

1. The patient underwent a minimum of eight (8) cold machine treatments but not to exceed a maximum of twelve (12) cold machine treatments

2. The patient had weight documentation before the cold machine treatment

3. The patient had weight documentation on a succeeding day after last treatment

\section{Exclusion criteria* for this study:}

1. Additional data in excess of 12 cold machine treatments will not be considered but the patient's first 12 treatments will still be used

2. Cold machine treatment schedule was done irregularly (more than 5 days based on computed "average days of interval") 
3. Cold wrap was done in between and/or during the cold machine treatments

4. Medical conditions that decrease the effect of any weight loss regimen including intake of medications that tend to increase weight

5. Going on any hypo caloric (crash) diet

6. Taking bariatric medications for the purpose of losing weight

* Inclusion/exclusion criteria are not the same as the indications and contraindications for cold machine treatment. Indications are overweight \& obese while contraindications are specific medical conditions.

\section{Results}

\section{Average weight loss per single treatment (by median)}

Highest weight loss+lowest weight loss $=1.4583$ lbs. $+(-0.44$ lbs. $=0.51 \mathrm{lbs}$.

\section{Average weight loss per single treatment (by mode)}

Given in table 1 (Table 1).

\section{Average weight loss per single treatment (by Mean)}

Sum of all weight loss $=415.65 \mathrm{lbs} .=0.4 \mathrm{lb} /$ treatment

Total No. of Treatments: 1,028 treatments

\section{Discussion}

First of all, this is not a prospective study wherein parameters were determined right at the start and was to be followed religiously. This is a retrospective study and inherently, some data as well as procedures may not be as standardized. Second, this is not an experimental cohort study wherein a "cohort" group would be treated with cold machine while another baseline group would be treated with the gold standard of the cold wraps and then compared. Nor is this an experimental casecontrol study wherein after certain patients lose weight (and/or inches), their centre treatments would be analysed to determine which possible centre treatment caused the weight loss (and/or inch loss). Rather this is a cross-sectional as well as longitudinal study of multiple case reporting wherein the patients' progress after being treated with cold machine were analysed and profile of the patients treated (as well as their results) were determined. Lastly, although there were so many variables that the investigator did not have control of since it is just a retrospective study, it is still considered a scientific study following all the principles involved in the scientific steps and processes.

At the start, the data gathering was initially straight-forward and contrite. At least that was the plan. I would be given access to the DCN (doctors, consultants, nutritionists) notes of all the centres and get my data from there. This proved to be challenging since data from there was sporadic. Certain technical issues regarding cross-branches also became apparent. The EDP (electronic data processing) was prone to

\begin{tabular}{|c|c|c|}
\hline \multicolumn{3}{|c|}{ Average weight loss per single treatment (by mode) } \\
\hline-0.49 to $-0.40 \mathrm{lb}=1$ & -0.00 to $0.09 \mathrm{lb}=3$ & $\mathbf{0 . 5 0}$ to $0.59 \mathrm{lb}=\mathbf{1 5}$ \\
\hline-0.39 to $-0.30 \mathrm{lb}=1$ & -0.10 to $0.19 \mathrm{lb}=13$ & 0.60 to $0.69 \mathrm{lb}=8$ \\
\hline-0.29 to $-0.20 \mathrm{lb}=2$ & 0.20 to $0.29 \mathrm{lb}=11$ & 0.70 to $0.79 \mathrm{lb}=3$ \\
\hline-0.19 to $-0.10 \mathrm{lb}=4$ & 0.30 to $0.39 \mathrm{lb} .=9$ & 0.80 to $0.89 \mathrm{lb}=7$ \\
\hline-0.09 to $-0.01 \mathrm{lb}=5$ & 0.40 to $0.49 \mathrm{lb}=16$ & 0.90 to $0.99 \mathrm{lb}=5$ \\
\hline & - & $1.0 \mathrm{lb}$ and above=3 \\
\hline
\end{tabular}

Table 1: Average weight loss per single treatment (by mode). errors and getting information that was needed became frustrating. So instead, ITS (Information Technology Department). Just gathered the data for me. This process was better as well as fast and systematic. Patients that underwent cold machine at least once in their lifetime in any of the centres were all collated together with their other treatments. And each treatment day had weight documentation specific per date. Results on weight loss per patient per centre were then easily computed, gathered, enumerated, and analysed.

But as other information was needed to paint a better and more complete picture, data gathering of additional miscellaneous pertinent data became a challenge. Since the population study including the actual "random sample population" was based on a master list of patients with cold machine treatments, the only identifying data are the patient's name, id number, and branch where he/she is based. From this list alone, data on weight loss documentation was already made easily. However, in order to identify the cross-section of the population, other data (like age, gender, and height) was needed. This is important to determine if there is a preference for success or failure based on age, gender, or height. Fortunately, this general data can be gathered from the hardcopy patient cards. There was also a need to look at medical doctor's notes and nutritionist's notes to complete the data. That is if the patient had unmanaged medical conditions or was doing a selfprescribed hypo caloric diet. Both would have disqualified the patient from being part of the sample population.

Then in proceeding with the longitudinal aspect of the study in comparing before and after inch loss, Health Risk Factor change, Body Fat Percentage change, Visceral Fat Rating change, and subcutaneous trunk fat change, body circumference measurements and TANITA readings were needed. These results had to be traced manually oneby-one in each slimming centre, including pertinent dates on when they were done (especially before and after the cold machine slimming program of 8-12 treatments within a regular schedule).

Regarding "randomness" in getting a representative "sample study population" for analysis, take a look at the data summary. Note that there's always at least one that gained weight (or considered an absolute failure with disappointing results) and there's also at least one that had a very large weight loss (or considered total success with miraculous results). This in itself is already a testament to its "randomness" since it manifested in all random samples per centre (Table 2). So it was inadvertently good that it's arranged the listing in alphabetical order of the first names of the patients (not surname). There was no predilection for gender since the listing did not list the females first and the males last, and/or vice-versa. There was no predilection for the date the patients were treated since it was not arranged chronologically (except per patient where the dates were arranged once the patient's name came up in the listing). There was no predilection either for age, weight, height, (nor race, skin colour, educational attainment, religion, etc). Since these data were not available in the master list of treatments. Only the names of the patient, the date of the treatment, the treatments done and weight documentations were the basis for the random sampling -plus the inclusion and exclusion criteria which they should qualify.

Regarding the BMI of the sample population (Table 3 and Figure 3), almost half were simply overweight while almost a quarter had normal weight, for a total of $72 \%$. For a slimming centre, one would assume that a large number of patients having weight loss programs would be those suffering from obesity. It also does not represent the typical general population where those with normal weight represent more than half and up to two-thirds of most populations in different countries [11]. At the same time, it should be noted that it was still ethical to treat those 
Citation: Torres AS (2017) The Effects of Cold Therapy in Losing Weight \& Inches as well as Decreasing Body Mass Index, Body Fat Percentage, and Lowering of Health Risk Factor. J Nutr Weight Loss 2: 107.

Page 4 of 8

\begin{tabular}{|c|c|c|c|c|c|c|c|c|c|c|}
\hline Centre E & Centre B & Centre $\mathbf{Q}$ & Centre P & Centre $\mathbf{F}$ & Centre Co & Centre Ce & Centre A & Centre T & Centre $\mathbf{O}$ & Centre $\mathbf{M}$ \\
\hline 0.483 & $\mathbf{1 . 0 2 5}$ & $\mathbf{- 0 . 0 5}$ & 0.4375 & 0.4 & $\mathbf{0 . 8 8 3 3}$ & 0.325 & 0.333 & 0.1833 & 0.475 & 0.1 \\
\hline 0.65 & 0.38 & 0.6 & $\mathbf{0 . 0 7 1 4}$ & 0.25 & $\mathbf{- 0 . 0 2 9}$ & 0.5625 & $\mathbf{0 . 7 7 5}$ & 0.15 & 0.182 & 0.08 \\
\hline $\mathbf{0 . 8}$ & 0.575 & $\mathbf{1 . 2 2}$ & 0.5 & $\mathbf{0 . 8 1 6 7}$ & 0.3333 & $\mathbf{- 0 . 0 2 5}$ & $\mathbf{- 0 . 0 5}$ & 0.2333 & 0.1 & 0.2333 \\
\hline 0.466 & 0.433 & $\mathbf{0 . 9 0 6 2 5}$ & 0.15625 & 0.425 & 0.2813 & 0.25 & 0.983 & 0.25 & 0.4 & $\mathbf{- 0 . 1 1 7}$ \\
\hline 0.483 & $\mathbf{0 . 8 7 5}$ & 0.5 & 0.5 & 0.3091 & 0.5 & 0.1 & $\mathbf{- 0 . 4 4}$ & 0.1 & 0.3 & $\mathbf{- 0 . 1 5}$ \\
\hline 0.636 & 0.533 & 0.367 & 0.9 & 0.402 & 0.42 & 0.55 & 0.18 & 0.16 & 0.2 & 0.15 \\
\hline 0.74 & 0.56 & 0 & 0.625 & 0.25 & 0.5208 & 0.5 & 0.22 & 0.16 & $\mathbf{- 0 . 0 3 6}$ & 0.525 \\
\hline 0.617 & 0.48 & None & 0.625 & $\mathbf{0 . 8 8 3 3}$ & 0.425 & 0.5 & 0.175 & 0.05 & 0.275 & $\mathbf{0 . 6 6 6 7}$ \\
\hline $\mathbf{- 0 . 2 5}$ & 0.8 & None & $\mathbf{- 0 . 1 2 5}$ & 0.6 & 0.42 & 0.5 & 0.45 & $\mathbf{1 . 4 5 8 3}$ & 0.45 & 0.2167 \\
\hline $\mathbf{0 . 9 2 7}$ & $\mathbf{- 0 . 1 5}$ & None & $\mathbf{0 . 9 3 7 5}$ & $\mathbf{- 0 . 2 2 5}$ & 0.5938 & None & 0.3167 & $\mathbf{- 0 . 3}$ & 0.382 & 0.85 \\
\hline
\end{tabular}

Table 2: Average weight loss per single treatment per center, (bold fonts mean weight gain and tremendous weight loss).

\begin{tabular}{|c|c|c|}
\hline $\mathbf{N}$ & 24 & $23 \%$ \\
\hline Overweight & 51 & $49 \%$ \\
\hline Obese I & 22 & $21 \%$ \\
\hline Obese II & 6 & $6 \%$ \\
\hline Obese III & 2 & $2 \%$ \\
\hline Total & 105 & $100 \%$ \\
\hline
\end{tabular}

Table 3: BMI classification change.

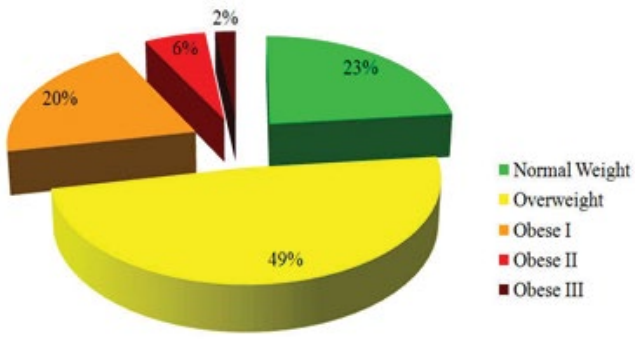

BMI Classification (based on WHO)

Figure 3: BMI classification based on WHO

with normal weight with a weight loss program if they are in the upper limit of the normal range.

Regarding the methodology of using 8-12 treatments to be considered a slimming program in the inclusion and exclusion criteria, the explanation put forth was based on clinical experience in the last 2 decades. In a previous unpublished in-house study using cold wraps done in 1998 in the same slimming centre (Figure 4), the best results "per treatment" were seen after 10 treatments $\left(6^{\text {th }}-10^{\text {th }}\right.$ or during the $2^{\text {nd }} 5$ treatments $)$ and after 15 treatments $\left(11^{\text {th }}-15^{\text {th }}\right.$ or during the $3^{\text {rd }} 5$ treatments) with only good results noted after 5 treatments $\left(1^{\text {st }}-5^{\text {th }}\right.$ or during the $1^{\text {st }} 5$ treatments). The results after 20 treatments $\left(15^{\text {th }}-20^{\text {th }}\right.$ or during the $4^{\text {th }} 5$ treatments) were much lower and obviously the plateau already.

The average weight loss per cold machine treatment is half a pound (Figures 5,6 and Table 2) while the average inch loss per cold machine program consisting of 8-12 treatments is one \& a half inches (Table 5 and Figure 7). For the Body Mass Index, there were those whose classifications were decreased from obese to overweight and from overweight to normal weight (Table 4). For the Health Risk Factor 9,10 (of developing heart attack and/or stroke), there were those who were Very High Risk that became Medium Risk and those who were Medium Risk that became Very Low Risk (Tables 6, 7 and Figure 8). For the Body Fat Percentages using the TINITA impedance machine, 31\%

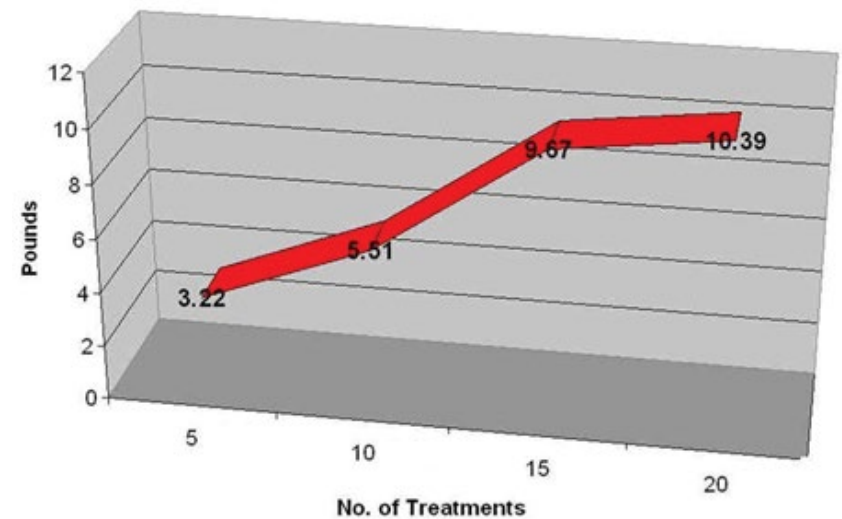

Figure 4: Results of Cold Wrap study done in 1998 after 5, 10, 15, 20 treatments.

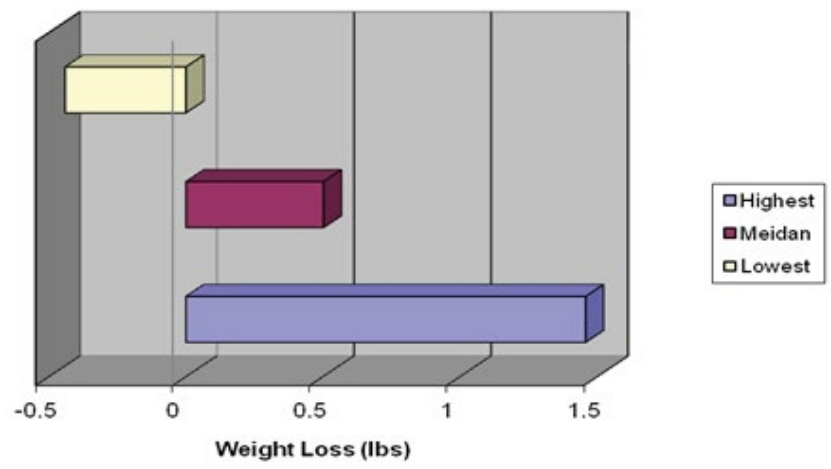

Figure 5: Average weight loss per single treatment (by Median).

decreased their Visceral Fat Rating and at the same time 77\% decreased the Subcutaneous Fat Volume in their tummy area. The same $77 \%$ also decreased their total Body Fat Percentage.

The result of the average weight loss by mode of $0.5 \mathrm{lb}$. (Figure 4) \& by median of $0.40-0.59 \mathrm{lb}$. \& $0.51 \mathrm{lb}$. (Figure 6) was good and satisfactory since it mimics the results of the previous study of the cold wraps of $0.5195 \mathrm{lb}$. However, it should be noted that the average weight loss per cold machine treatment by mean is $0.40 \mathrm{lb}$. (Table 2) and that 4 of the biggest centres (out of 11) are below this and even represent the 4 least results. It is these results that pulled the mean average down since the other 7 centres had results at approximately $0.5 \mathrm{lb}$. per cold machine treatment. Similarly, the failure rate per centre (based on weight gain 
Citation: Torres AS (2017) The Effects of Cold Therapy in Losing Weight \& Inches as well as Decreasing Body Mass Index, Body Fat Percentage, and Lowering of Health Risk Factor. J Nutr Weight Loss 2: 107.

Page 5 of 8

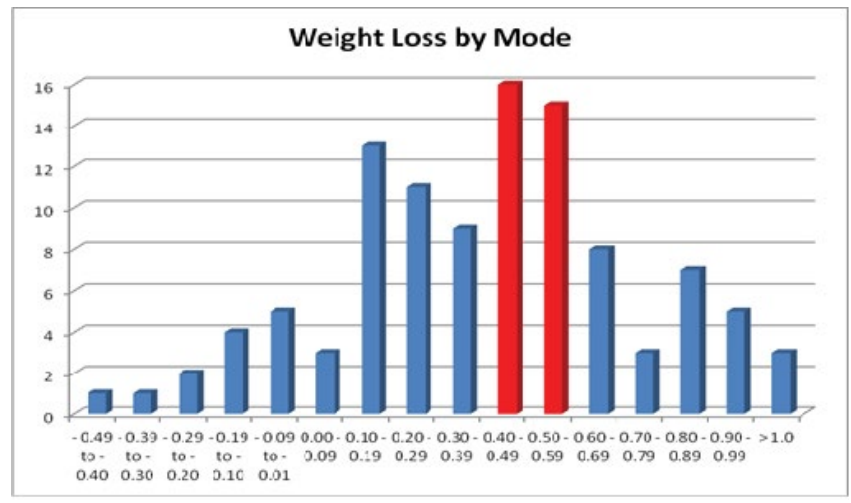

Figure 6: Average weight loss per single treatment (by Mode).

\begin{tabular}{|c|c|c|c|}
\hline Branch & $\begin{array}{c}\text { BMI Class } \\
\text { Improved }\end{array}$ & $\begin{array}{c}\text { BMI Class Not } \\
\text { Improved }\end{array}$ & Total \\
\hline Center E & 1 & 9 & 10 \\
\hline Center B & 0 & 10 & 10 \\
\hline Center Co & 4 & 6 & 10 \\
\hline Center P & 4 & 6 & 10 \\
\hline Center Q & 1 & 5 & 6 \\
\hline Center F & 1 & 9 & 10 \\
\hline Center Ce & 1 & 8 & 9 \\
\hline Center T & 0 & 10 & 10 \\
\hline Center M & 1 & 9 & 10 \\
\hline Center A & 0 & 10 & 10 \\
\hline Center O & 2 & 8 & 10 \\
\hline TOTAL & $\mathbf{1 5}$ & $\mathbf{9 0}$ & $\mathbf{1 0 5}$ \\
\hline
\end{tabular}

Table 4: Body mass index cross-section.

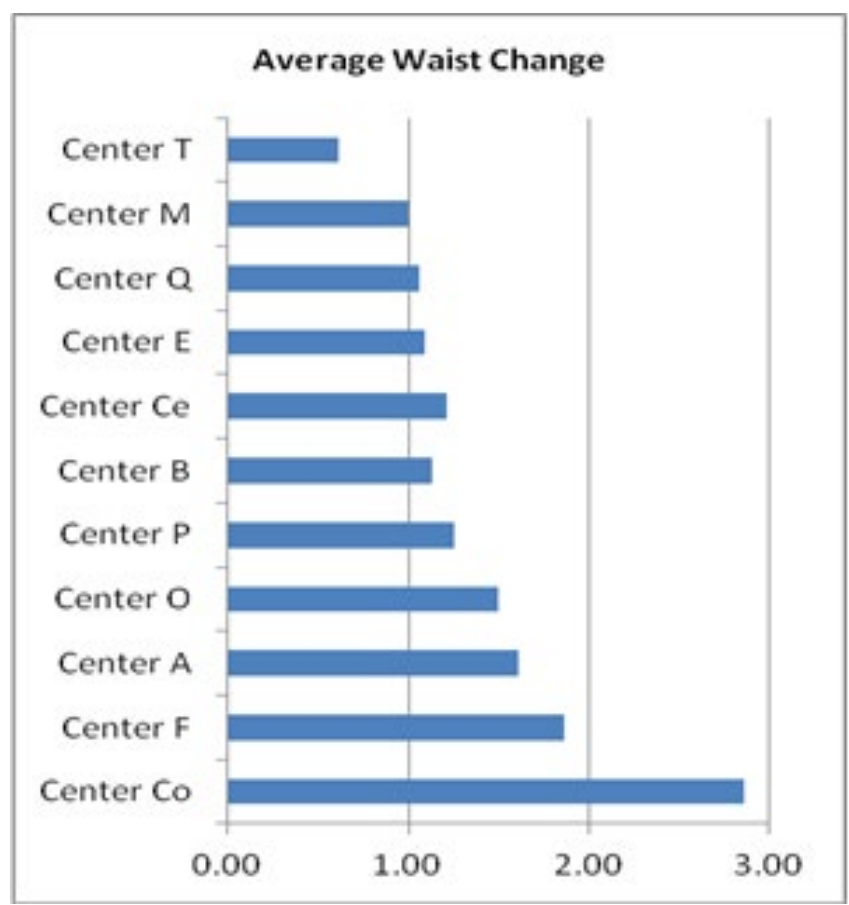

Figure 7: Average waist change.

\begin{tabular}{|c|c|}
\hline Branch & Average Waist Change \\
\hline Centre CO & 2.86 \\
\hline Centre $\mathrm{F}$ & 1.86 \\
\hline Centre A & 1.61 \\
\hline Centre O & 1.50 \\
\hline Centre P & 1.25 \\
\hline Centre B & 1.13 \\
\hline Centre Ce & 1.21 \\
\hline Centre E & 1.09 \\
\hline Centre Q & 1.6 \\
\hline Centre M & 1.00 \\
\hline Centre T & 1.61 \\
\hline Average & 1.52 inches \\
\hline
\end{tabular}

Table 5: Inch loss

\begin{tabular}{|c|c|c|c|c|}
\hline \multirow{2}{*}{\multicolumn{2}{|c|}{ Females }} & \multicolumn{3}{|c|}{ Waist Circumference } \\
\hline & & $<27 \frac{1}{2}$ inches & $\begin{array}{c}27 \frac{1}{2}-341 / 2 \\
\text { inches }\end{array}$ & $>341 / 2$ inches \\
\hline \multicolumn{2}{|c|}{ Males } & $<31 \frac{1}{2}$ inches & $31 \frac{1}{2}-40$ inches & $>40$ inches \\
\hline \multicolumn{2}{|c|}{ Body Mass Index } & \multirow{2}{*}{\multicolumn{3}{|c|}{ - }} \\
\hline Classification & BMI Range & & & \\
\hline Normal Weight & $18.5-24.9$ & Very Low Risk & Low Risk & Medium Risk \\
\hline Overweight & $25.0-29.9$ & Low Risk & Medium Risk & High Risk \\
\hline Obese I & $30.0-34.9$ & Medium Risk & High Risk & Very High Risk \\
\hline Obese II & $35.0-39.9$ & High Risk & Very High Risk & Very High Risk \\
\hline Obese III & $\geq 40.0$ & $\begin{array}{c}\text { Moderately High } \\
\text { Risk }\end{array}$ & Very High Risk & Very High Risk \\
\hline
\end{tabular}

Table 6: Health risk stratification.

\begin{tabular}{|l|c|}
\hline \multicolumn{1}{|c|}{ Branch } & Health Risk Change \\
\hline Center E & of 8 \\
\hline Center B & 1 of 8 \\
\hline Center Q & 2 of 4 \\
\hline Center P & 5 of 7 \\
\hline Center F & 5 of 9 \\
\hline Center Co & 7 of 9 \\
\hline Center Ce & 3 of 7 \\
\hline Center A & 0 of 9 \\
\hline Center T & 0 of 9 \\
\hline Center O & 4 of 10 \\
\hline Center M & 1 of 5 \\
\hline TOTAL & 29 of 90 \\
\hline
\end{tabular}

Table 7: Health risk change.

Health Risk Change

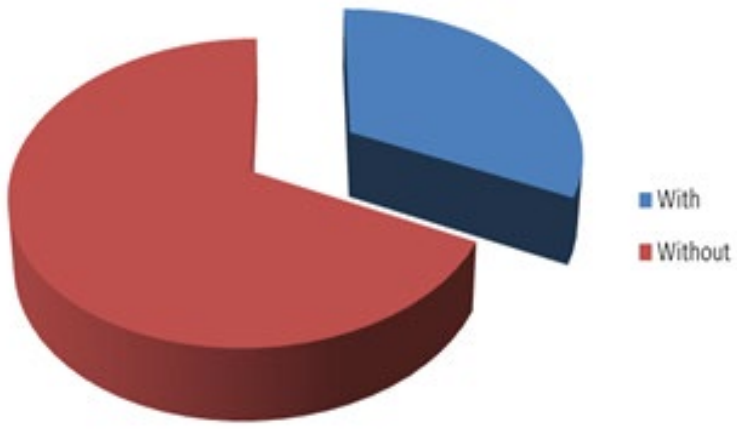

Figure 8: Health risk change. 
Citation: Torres AS (2017) The Effects of Cold Therapy in Losing Weight \& Inches as well as Decreasing Body Mass Index, Body Fat Percentage, and Lowering of Health Risk Factor. J Nutr Weight Loss 2: 107.

Page 6 of 8

instead of weight loss) is $10 \%$ which is also statistically acceptable and a testament to the randomness of the sample population. But not in centre $\mathrm{A}$ and centre $\mathrm{M}$ where it is $20 \%$ (Table 2).

The result of the average inch loss after a slimming program consisting of $8-12$ cold machine treatments of $1 \frac{1 / 2}{2}$ inches was good. However, the extreme results of the centre with the highest inch loss (2.86 inches) and the centre with the lowest inch loss (0.6 inches) should be noted. Explanations on why this happened should be taken into consideration for standardization purposes as well as for future studies. Regarding the "big divide" between the results of the number of patients with a decrease in total Body Fat Percentage and Subcutaneous Fat Volume (both at $77 \%$ and representing the same patients) versus the number of patients with a decrease in Visceral Fat Rating (only at $31 \%$ ), this can be easily explained. Based on experience coupled with the technology involved in the impedance method of taking Body Fat Percentages, it usually takes $10-15$ pounds of weight loss before the Visceral Fat Rating changes (Figures 9,10).

Although the male to female ratio for all the patients that underwent cold machine treatments was $1: 10$, it should be noted that the male to female ratio in the random sampling (excluding $\mathrm{Q}$ whose patients are purely male) was $1: 4$. This may mean that male patients are more obedient in following the correct protocol for cold machine which is to do it regularly 2-3x a week, and continuously for 2-3 months [Figures 11 and 12]. That is why after applying the inclusion and exclusion criteria, most of the male patients qualified as compared to their female counterparts. Therefore, the only reason that the female patients still predominated is because they were more prominent in number from the start wherein females are more conscious about their weight and Figure to the point of going to a slimming centre for weight loss and inch loss intervention (Figures 1 and 2).

After careful analysis of the weight loss and inch loss results including the improvements in Health Risk Factor and body fat impedance (Body Fat Percentage, visceral fat, and trunk fat volume), the other accompanying machine treatments or absence thereof did not have a discernible correlation. There were some who did not have machine treatments yet resulted with better inch loss results. Similarly,

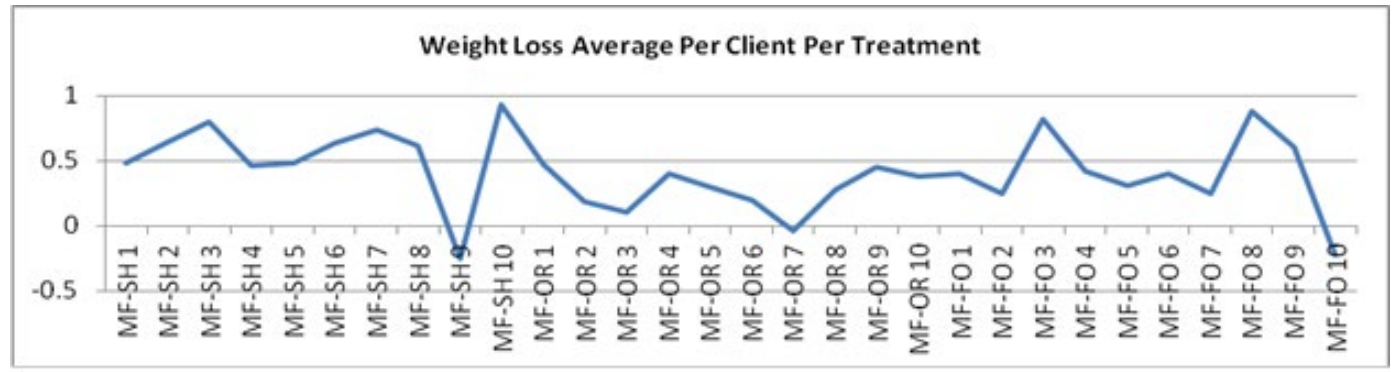

Figure 9: Line graphs showing trends of results (weight loss/weight gain) per 2-3 centres (centre S, centre O, centre F)

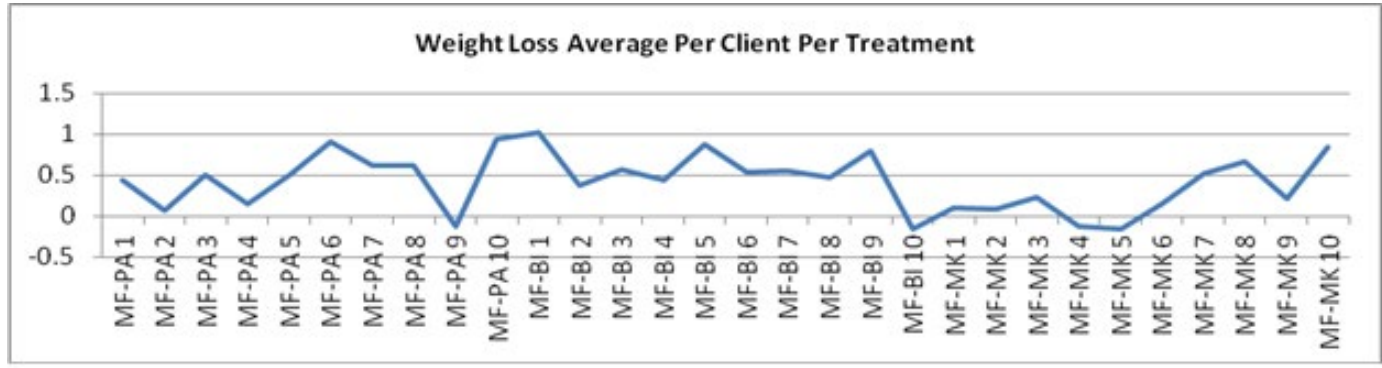

Figure 10: Weight loss average per client per treatment (centre $\mathrm{P}$, centre $\mathrm{B}$, centre $\mathrm{M}$ ).

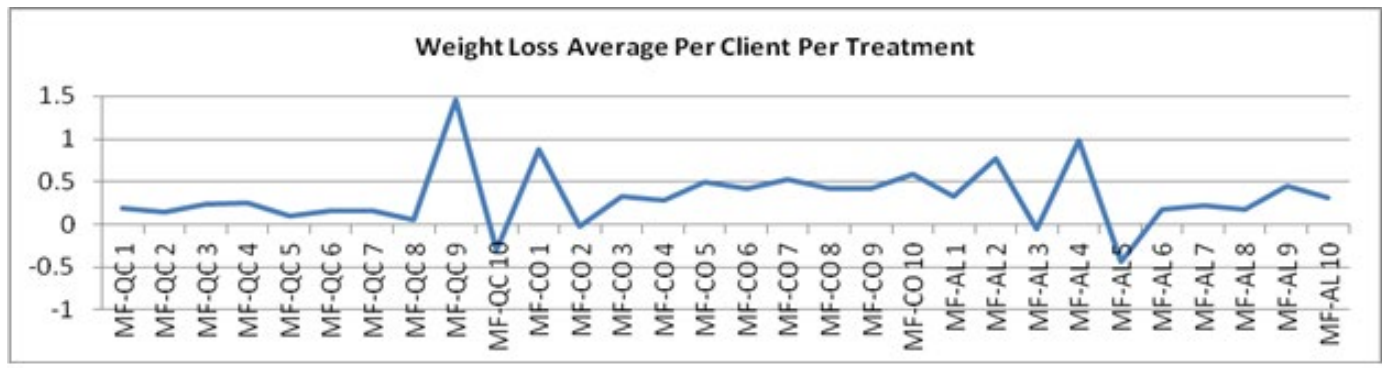

Figure 11: Weight loss average per client per treatment (centre $\mathrm{T}$, centre $\mathrm{CO}$, centre $\mathrm{A}$ ). 


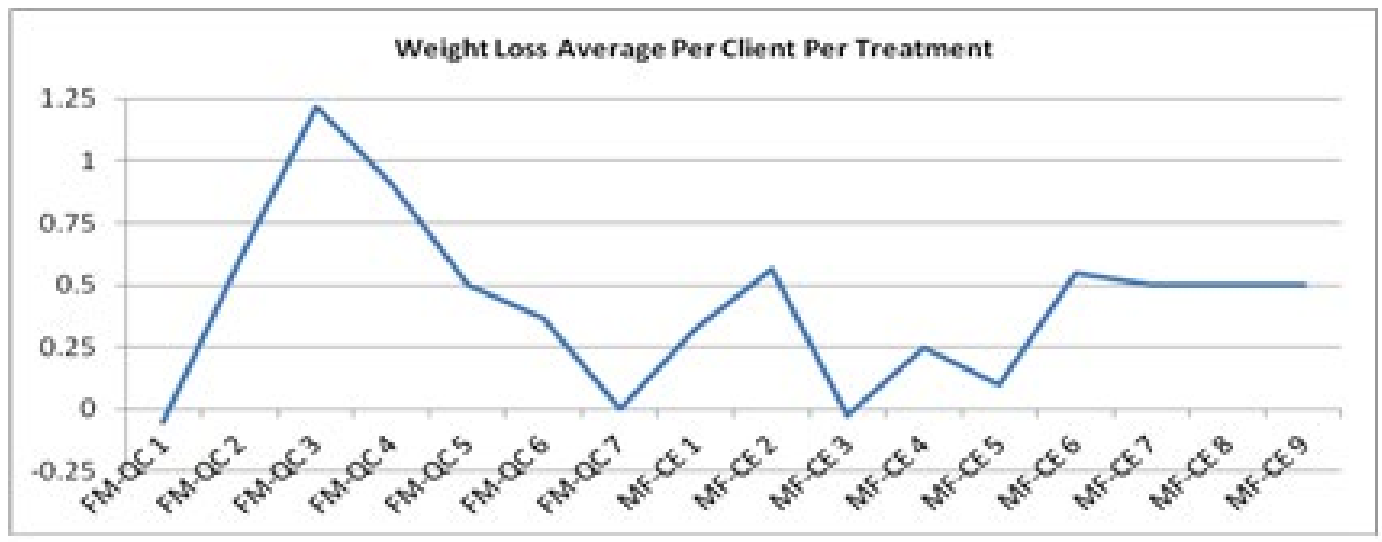

Figure 12: Weight loss average per client per treatment (centre $\mathrm{Q}$, centre $\mathrm{Ce}$ ).

there were some who had additional machine treatments but did not manifest with better weight loss results. Because of this, it was not included anymore in the data analysis and results although the treatments were enumerated at the table of sample patients included in the study per centre.

\section{Limitation of the Study}

As stated earlier, the patients in this study are private paying clients who went to the centre in order to lose weight and inches, as well as getting in shape not just for vanity but for health and wellness. So this is more of a multiple case report where all of the subjects underwent slimming programs consisting of the cold machine treatment and their average results tallied. A randomized controlled trial (RCT) would have been better wherein the study group would be given the cold therapy while the control group would be given a placebo therapy but both groups would be given the same advice on diet and activity. But the objective of the study was met wherein results of a similar slimming program using the old \& primitive cold wraps was being compared to the new innovation of using a cold machine treatment with a thermostat for a more controlled and uniform treatment. The messy and unpleasant feeling of being wet with the bandages soaked in solutions before has been replaced with a more comfortable system that also lessens the chances of superficial skin infections being introduced iatrogenic. Shivering was also dramatically decreased and even totally absent in others.

Not all had complete documentation like daily weight measurements and scheduled body composition analysis. Some patients opt to waive the procedure of being weighed in some days while others didn't care about having a Body Composition Analysis done. In some cases, they were simply missed and forgotten. Though these patients were disqualified based on the inclusion and exclusion criteria, it may have inadvertently affected the random sampling and confounded the results (e.g. those that didn't want to be weighed could be the ones with either better or lesser results).

Some of the sample population had other machine treatments for anti-cellulite as well as for firming and contouring of other body fat areas (arms, thighs) and therefore should not affect the results (visceral fat inside the tummy and subcutaneous fat on top of the tummy). These treatments also have no effect on weight loss or inch loss. However, their mere presence can be misconstrued as a confounding factor since they are still considered "slimming" treatments.
Although the nutrition and activity of the patients were monitored and taken into consideration, it would have been better if the subjects were observed in a more controlled environment. This is the major difference between "in vitro" or under the glass observations as compared to "in vivo" or in real living conditions. So even though the daily caloric allowance was computed based on the activity of the patient so as to make sure that their food intake is commensurate to what they are doing in order to simulate a homeostatic energy balance, the investigator had no control over the honesty as well as accurate reporting (food intake and physical exercise) of the subjects. That is the disadvantage but also the advantage since real life uncontrolled situations will be taken into account regarding the results.

\section{Summary and Conclusion}

Cold treatment using cold machine mimics the same approximate weight loss result of the cold wraps ( $0.5091 \mathrm{lb}$.vs. $0.5195 \mathrm{lb}$. respectively). It is an effective treatment for inducing weight loss and producing inch loss. Since the height of the patients do not change, the body mass index (BMI) which is computed using patient's weight and height also decreased. This resulted in some patients changing from a higher to a lower classification (from being obese to simply being overweight) in BMI classification. Since the BMI and waist circumference decreased (inch loss) at the same time, the Health Risk Factor classification also decreased (from high risk to low risk). Simultaneously, the Body Fat Percentage (whole body fat), visceral fat (intra-abdominal fat) and trunk fat (subcutaneous tummy fat) decreased after undergoing cold machine treatments.

\section{Recommendations}

The results of the Body Mass Index (BMI) changes, Health Risk Factor (HRF) changes, Body Fat Percentage (BFP) changes, Visceral Fat Rating (VFR) changes, including subcutaneous trunk fat (SCF) lowering are very promising and should be taken advantage of. Their health implications on improving the quality of life and even preserving it are of vital importance to some if not most of our patients. On top of that are the documented average weight loss of half a pound per cold machine treatment and inch loss of one \& a half inches per entire cold machine slimming program that can all be used to inform others. I therefore recommend that these data should be shared and coordinated with other health professionals, especially those involved with weight loss for their patients suffering from obesity where being overweight is a risk factor for metabolic as well as cardiovascular diseases. 
Citation: Torres AS (2017) The Effects of Cold Therapy in Losing Weight \& Inches as well as Decreasing Body Mass Index, Body Fat Percentage, and Lowering of Health Risk Factor. J Nutr Weight Loss 2: 107.

\section{References}

1. Van A, Hoeks J, Brans B, Vijgen G, Visser M, et al. (2013) Cold acclimation recruits human brown fat and increases non-shivering thermogenesis. $\mathrm{J}$ Clin Invest 8: 3395-3403.

2. Davis T (1961) Chamber cold acclimatization in man. Journal of applied physiology 6: 1011-1015.

3. Saito M, Okamatsu Y, Matsushita M, Watanabe K, Yoneshiro T, et al. (2009) High incidence of metabolically active brown adipose tissue in healthy adult humans-effects of cold exposure and adiposity. Diabetes 58: 1526-1531.

4. Cannon B, Nedergaad J (2004) Brown adipose tissue: Function and physiologic significance. Physiol Rev 84: 277-359.

5. Watanabe M, Yamamoto T, Mori C, Okaida N, Yamazaki N, et al. (2008) Coldinduced changes in gene expression in BAT: Implications for the activation of thermogenesis. Biol Pharm Bull 31(5) 775-784

6. Grio R, Porpiqlia M (1994) Efficacy of fat mobilisation system (cold wrap) in the treatment of obesity and its utility in the resolution of gynecological problems related to overweight. Panminerva Medica 36: 142-148.

7. Dasgupta S, Hazra SC (1999) The utility of waist circumference in assessment of obesity. Indian J Public Health 43: 132-135.

8. Ross R, Rissanen J, Hudson R (1996) Sensitivity associated with the identification of visceral adipose tissue levels using waist circumference in men and women: Effect of weight loss. International J of Obes 20: 533-538.

9. Zhou BF (2002) Predictive values of body mass index and waist circumference for risk factors of certain related diseases in chinese adults. Biomedical and Environ Sci 15: 83-96.

10. Reeder BA, Senthilselvan A, Despres JP, Angel A, Liu L, et al. (1997) The association of cardiovascular disease risk factors with abdominal obesity in canada. CMAJ 157: S39-S45.

11. NCD risk factor collaboration (2016) Trends in adult body mass index in 200 countries from 1975 to 2014. The Lancet 387: 1377-1396. 